\title{
Superconductivity on $\mathrm{ScH}_{3}$ and $\mathrm{YH}_{3}$ hydrides: Effects of applied pressure in combination with electron- and hole-doping on the electron-phonon coupling properties
}

\author{
S. Villa-Cortés ${ }^{1, *}$ and O. De la Peña-Seaman ${ }^{1}$ \\ ${ }^{1}$ Instituto de Física, Benemérita Universidad Autónoma de Puebla, \\ Apartado Postal J-48, 72570, Puebla, Puebla, México
}

(Dated: December 21, 2021)

\begin{abstract}
The implementation of electron- and hole-doping, in conjunction to applied pressure, is analyzed as a mechanism to induce or enhance the superconducting state on fcc $\mathrm{YH}_{3}$ and $\mathrm{ScH}_{3}$. In particular, the evolution of their structural, electronic, and lattice dynamical properties, as well as the electron-phonon coupling and superconducting critical temperature $\left(T_{c}\right)$ is presented and discussed, as a function of the electron- and hole-doping content as well as applied pressure. The study was performed within the density functional perturbation theory, taking into account the effects of zero-point energy through the quasi-harmonic approximation, while the doping was implemented by means of the construction of the $\mathrm{Sc}_{1-x} \mathrm{M}_{x} \mathrm{H}_{3}(\mathrm{M}=\mathrm{Ca}, \mathrm{Ti})$ and $\mathrm{Y}_{1-x} \mathrm{M}_{x} \mathrm{H}_{3}(\mathrm{M}=\mathrm{Sr}, \mathrm{Zr})$ solid solutions modeled with the virtual crystal approximation (VCA). We found that the $\mathrm{ScH}_{3}$ and $\mathrm{YH}_{3}$ hydrides shown a significant improvement of their electron-phonon coupling properties under hole-doping $(\mathrm{M}=\mathrm{Ca}, \mathrm{Sr})$ and at pressure values close to dynamical instabilities. Instead, by electron-doping $(\mathrm{M}=\mathrm{Ti}, \mathrm{Zr})$, the systems do not improve such properties, whatever value of applied pressure is considered. Then, as a result, $T_{c}$ rapidly increases as a function of $x$ on the hole-doping region, reaching its maximum value of $92.7(67.9) \mathrm{K}$ and $84.5(60.2) \mathrm{K}$ at $x=0.3$ for $\mathrm{Sc}_{1-x} \mathrm{Ca}_{x} \mathrm{H}_{3}$ at $10.8 \mathrm{GPa}$ and $\mathrm{Y}_{1-x} \mathrm{Sr}_{x} \mathrm{H}_{3}$ at $5.8 \mathrm{GPa}$ respectively, with $\mu^{*}=0(0.15)$, while for both, electron- and hole-doping, $T_{c}$ decreases as a function of the applied pressure, mainly due to phonon hardening. By the thorough analysis of the electron-phonon properties as a function of doping and pressure, we can conclude that the tuning of the lattice dynamics is a promising path for improving the superconductivity on both systems.
\end{abstract}

\section{INTRODUCTION}

Materials with a high-superconducting critical temperature $\left(T_{c}\right)$ are of great interest since at ambient conditions could have many technological applications. The theoretical breakthrough and progress for reaching high- $T_{c}$ superconductivity came after the Ashcroft[1] pioneering idea, suggesting that hydrogen-rich materials could be promising candidates for high- $T_{c}$ superconductivity. After that, several theoretical predictions have been proposed and performed on the crystal structure, at high pressure, of stoichiometric and hydrogen-rich materials, for which their electronic structure, lattice dynamics, and electron-phonon (el-ph) coupling properties have been calculated[2-5]. As a result of those predictions, several metal hydrides were proposed as conventional-superconductor candidates with a $T_{c}$ near room-temperature[6-8]. The experimental breakthrough came with the discovery of phonon-mediated superconductivity on $\mathrm{H}_{3} \mathrm{~S}$, with a $T_{c}$ of $203 \mathrm{~K}$ under pressures as high as $155 \mathrm{GPa}[9,10]$. Some years latter, high- $T_{c}$ superconductivity measurements were reported in other compounds, all of them at high applied pressure values, like $\mathrm{LaH}_{10}$ with $T_{c}$ in the range of 250-260 K[11, 12] at $170 \mathrm{GPa}$, and more recently, $\mathrm{YH}_{9}$ with $T_{c}=262 \mathrm{~K}$ at $182 \mathrm{GPa}[13]$ and $\mathrm{YH}_{6}$ giving $T_{c}=220 \mathrm{~K}$ at $183 \mathrm{GPa}[14]$,

* svilla@ifuap.buap.mx as well as a top reported $T_{c}$ value of $287 \mathrm{~K}$ in a carbonaceous sulfur-hydride at $267 \mathrm{GPa}[15]$.

As a result of the available theoretical and experimental reports, it has been determined that the tendency for superconductivity depends upon the species used to build up the metal hydride (together with hydrogen), a high density of states at the Fermi level, and that the resulting hydride compound must have a large electronphonon coupling related to the hydrogen atoms. In particular, some of the highest $T_{c}$ values have being obtained from hydrides constructed with elements that belong to the alkaline family as well as the scandium group (first group of the transition metals) [5]. Regarding this group, from calculations on $\mathrm{YH}_{n}$, the $T_{c}$ was estimated around $305-326 \mathrm{~K}$ at an applied pressure of $250 \mathrm{GPa}$ for $n=10$ $[5,7]$; while for $n=6, T_{c}$ was on the range of $251-264 \mathrm{~K}$ at $120 \mathrm{GPa}[5]$; and for $n=4$, lower $T_{c}$ values (around $84-95 \mathrm{~K}$ ) at $120 \mathrm{GPa}$ were reported [16]. For this family, only the $\mathrm{YH}_{9}$ and $\mathrm{YH}_{6}$ have been studied experimentally, as already mentioned. For the $\mathrm{ScH}_{n}$ family, $T_{c}$ in the range of 120-169 $\mathrm{K}$ was predicted for different members, with $n=6,7,9,10$ and 12 , for an applied pressure above $250 \mathrm{GPa}[5,17]$. All the already mentioned metal hydrides can be considered as chemically precompressed phases relative to pure hydrogen, where high pressure is necessary for metallization[1].

With respect to $\mathrm{ScH}_{3}$ and $\mathrm{YH}_{3}$ (metal-hydrides with low hydrogen content), they have hcp structures at ambient pressure, and are driven to fcc (cubic $\mathrm{NaCl}$ (B1) structure) (see Fig.1) phase under applied pressure. For 
$\mathrm{YH}_{3}$, Raman [18] and infrared [19] studies found that the cubic structure can exist at approximately $10 \mathrm{GPa}$, and is clearly stabilized around $25 \mathrm{GPa}$. It has been suggested that another intermediate phase [20] or a coexisting hcpcubic phase [21] could also appear in the 8-25 GPa pressure range. It has been shown experimentally that $\mathrm{YH}_{3}$ can be stabilized in the fcc phase at ambient pressure by substituting $\mathrm{Y}$ for $10 \% \mathrm{Li}\left(\mathrm{Li}_{0.1} \mathrm{Y}_{0.9} \mathrm{H}_{3}\right)[22,23]$. Recently, J. Purans et al.[24] and Kong et al.[14] reported the syntheses of this metal-hydride with a pure metallic fcc phase at a broad pressure interval, $40-340 \mathrm{GPa}$. For lower applied pressure values, between 20 and $40 \mathrm{GPa}$, they found it to be a semi-metal with a distorted fcc crystal structure. Similarly, for $\mathrm{ScH}_{3}$, Raman and infrared[25] studies observed an hcp-intermediate-cubic phase at $25 \mathrm{GPa}$. Theoretically, Kong et al.[26] reported the hcp-cubic phase transition at $25 \mathrm{GPa}$, while Pakornchote et al.[27] found the cubic phase to be energetically more stable at slightly lower pressure of $22 \mathrm{GPa}$. For these metalhydrides, $\mathrm{YH}_{3}$ and $\mathrm{ScH}_{3}$, Kim et al.[5, 28] performed first principles calculations and found that the fcc structure is dynamically stable on both of them at a pressure of $\approx 18 \mathrm{GPa}$, with an estimated $T_{c}$ around 18 and $40 \mathrm{~K}$, respectively, with a rapid decreased of $T_{c}$ as the pressure is incremented, in agreement with the experimental report of Kong et al.[14] where superconductivity where not found above $5 \mathrm{~K}$ for metallic fcc structure.

Besides applied pressure, doping is another mechanism for metallization of metal hydrides, in order to induce or increase superconductivity by the improvement of some properties, like the electronic density of states at the Fermi level $(N(0))$ or the el-ph coupling. For example, the substitution of $\mathrm{Li}$ by $\mathrm{Be}, \mathrm{Mg}$, or $\mathrm{Ca}$ in $\mathrm{LiH}$ was studied by Zhang et al.[29]. In that work, the dopant acts as a donor which delivers electrons to the system, obtaining a $n$-doped material with a $T_{c}=7.78 \mathrm{~K}$ for an electron content as high as 2.06, calculated at ambient pressure. Another work on that direction was performed by Olea-Amezcua et al.[30]. There the authors showed the metallization of alkali-metal hydrides $\mathrm{LiH}, \mathrm{NaH}$, and $\mathrm{KH}$ by doping with alkaline-earth metals $\mathrm{Be}, \mathrm{Mg}$, and $\mathrm{Ca}$, respectively, and analyzed the superconducting properties as a function of metal content. The maximum estimated $T_{c}$ values were $2.1 \mathrm{~K}$ for $\mathrm{Li}_{0.95} \mathrm{Be}_{0.05} \mathrm{H}, 28 \mathrm{~K}$ for $\mathrm{Na}_{0.8} \mathrm{Mg}_{0.2} \mathrm{H}$, and even $49 \mathrm{~K}$ for $\mathrm{K}_{0.55} \mathrm{Ca}_{0.45} \mathrm{H}$, without applied pressure. More recently, Villa-Cortés et al. [31] studied the structural, electronic, and lattice dynamical properties, as well as the electron-phonon coupling and superconducting critical temperature $\left(T_{c}\right)$ of $\mathrm{ScH}_{2}$ and $\mathrm{YH}_{2}$ metal hydrides solid solutions, as a function of the electron- and hole-doping content, in absence of applied pressure. They found that for electron-doping content $x>0.5, T_{c}$ increases rapidly, reaching its maximum value of the entire range at the $\mathrm{Sc}_{0.05} \mathrm{Ti}_{0.95} \mathrm{H}_{2}$ and $\mathrm{Y}_{0.2} \mathrm{Zr}_{0.8} \mathrm{H}_{2}$ solid solutions. These results show that such scheme to induce metallization and superconductivity on metal-hydrides works as an alternative to the appliedpressure approach. So, in this paper we implement it, in

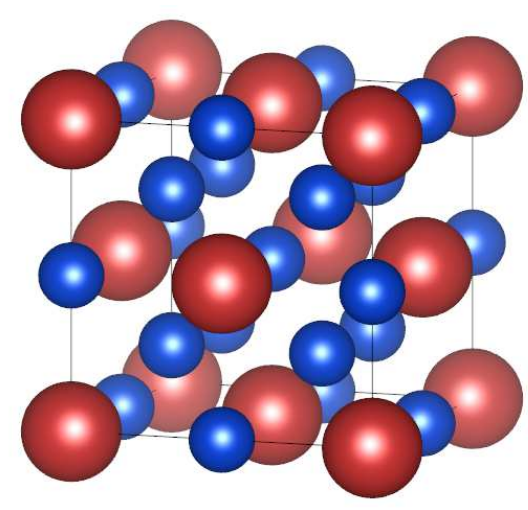

FIG. 1. Fcc (cubic NaCl (B1)) structure (space group $F m \overline{3} m$ ) of the $\mathrm{Sc}_{1-x} \mathrm{M}_{x} \mathrm{H}_{3}$ and $\mathrm{Y}_{1-x} \mathrm{M}_{x} \mathrm{H}_{3}$ solid solutions. The Scandium(Yttrium) and Hydrogen atoms are represented by large red and small blue spheres, respectively.

addition to applied pressure, in order to study the $\mathrm{ScH}_{3}$ and $\mathrm{YH}_{3}$ compounds in the fcc structure $(\mathrm{NaCl}(\mathrm{B} 1)$ phase), which is reported to present superconductivity, as discussed previously. Under this approach we are able to trace down the evolution of the structural, electronic, and lattice dynamical properties, as well as the el-ph coupling and $T_{c}$, as a function of metal content, by inducing holes ( $p$-doped) and electrons ( $n$-doped), as well as applied pressure, of the proposed systems. Such approach is done by the construction of solid solutions with the metal atom of the hydride: $\mathrm{Sc}_{1-x} \mathrm{M}_{x} \mathrm{H}_{3}(\mathrm{M}=\mathrm{Ca}, \mathrm{Ti})$ and $\mathrm{Y}_{1-x} \mathrm{M}_{x} \mathrm{H}_{3} \quad(M=\mathrm{Sr}, \mathrm{Zr})$ within the Density Functional Theory (DFT)[32], using the virtual crystal approximation (VCA)[33], which has been successfully applied on the study of doped superconductors [30, 34-37].

The paper is organized as follows. The computational details of our method are presented in Section II. In Section III.A we present our results related to the structural properties; while in Section III.B the electronic structure analysis is shown. The lattice dynamics are discussed in Section III.C; and the electron-phonon and superconducting properties, as well as $T_{c}$, are shown in Section III.D. Last, our conclusions are presented in Section IV.

\section{COMPUTATIONAL DETAILS}

The calculations of the structural, electronic, lattice dynamics and el-ph coupling properties were carried out within the framework of Density Functional Theory (DFT) [32] and Density Functional Perturbation Theory (DFPT) [38, 39], both implemented in the QUANTUM ESPRESSO suit code [38]. The calculations were performed with a $24 \times 24 \times 24 k$-point mesh, and a $60 \mathrm{Ry}$ cutoff for the plane-wave basis, while the Perdew-BurkeErnzerhof (PBE) functional [40] was employed to take into account the exchange and correlation contributions.

Fourier interpolation of dynamical matrices, calculated on a $8 \times 8 \times 8 q$-point mesh, were used to de- 

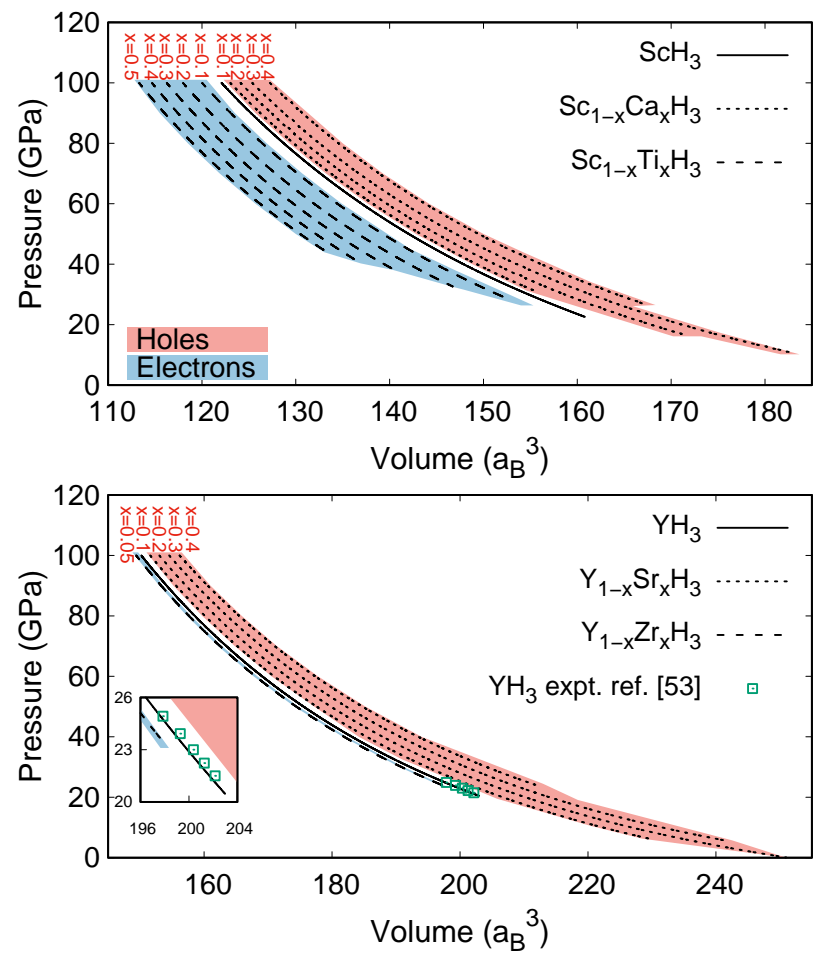

FIG. 2. $P V$ equation of state of $\mathrm{Sc}_{1-x} \mathrm{M}_{x} \mathrm{H}_{3}$ and $\mathrm{Y}_{1-x} \mathrm{M}_{x} \mathrm{H}_{3}$, for different metal $\mathrm{M}$ content $(x)$, studied within the ZPE scheme. $a_{B}$ stands for the Bohr radius.

termine the complete phonon spectra of the studied systems. Corrections due to quantum fluctuations at zero temperature, zero-point energy (ZPE) effects, are estimated through the quasi-harmonic approximation (QHA)[30, 41]. Within this approximation, the phonon contribution to the ground-state energy is taken into account and then a equation of state for each concentration $x$ can be constructed. Thus, the electronic structure, lattice dynamics, and el-ph properties, calculated for the fcc (B1) crystal structure at different applied pressure values, incorporate the ZPE correction.

Included on the el-ph calculations, the phonon linewidths of the $\vec{q} \nu$ phonon mode $\gamma_{\vec{q} \nu}$ were also obtained, which are given by $[42,43]$

$\gamma_{\vec{q} \nu}=2 \pi \omega_{\vec{q} \nu} \sum_{\vec{k} n m}\left|g_{\vec{k}+\vec{q}, \vec{k}}^{\vec{q} \nu, n m}\right|^{2} \delta\left(\epsilon_{\vec{k}+\vec{q}, m}-\epsilon_{F}\right) \delta\left(\epsilon_{\vec{k}, n}-\epsilon_{F}\right)$,

where $g_{\vec{k}+\vec{q}, \vec{k}}^{\vec{q} \nu, n m}$ are the matrix elements of the electronphonon interaction (calculated over a denser $48 \times 48 \times$ $48 k$-point mesh), $\epsilon_{\vec{k}+\vec{q}, m}$ and $\epsilon_{\vec{k}, n}$ are one-electron band energies, with band index $m, n$, and vectors $\vec{k}+\vec{q}, \vec{k}$, respectively, while $\omega_{\vec{q} \nu}$ is the phonon frequency for mode $\nu$ at wave-vector $\vec{q}$.

With the knowledge of $\gamma_{\vec{q} \nu}$ and $\omega_{\vec{q} \nu}$, the isotropic Eliashberg spectral function, $\alpha^{2} F(\omega)$, can be described as

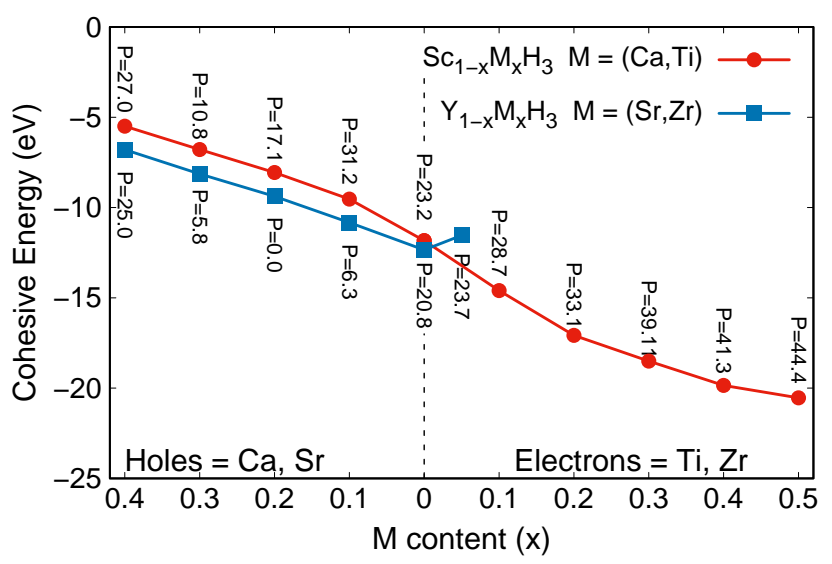

FIG. 3. Cohesive energy of $\mathrm{Sc}_{1-x} \mathrm{M}_{x} \mathrm{H}_{3}$ and $\mathrm{Y}_{1-x} \mathrm{M}_{x} \mathrm{H}_{3}$ as a function of the metal $\mathrm{M}$ content $(x)$ for the minimum pressure value in the stability range of each $x$.

$$
\alpha^{2} F(\omega)=\frac{1}{2 \pi \hbar N(0)} \sum_{\vec{q} \nu} \delta\left(\omega-\omega_{\vec{q} \nu}\right) \frac{\gamma_{\vec{q} \nu}}{\omega_{\vec{q} \nu}}
$$

where $N(0)$ is the electronic density of states (per atom and spin) at $\epsilon_{F}$, and a sum over a denser Fourier interpolated $q$-point mesh of $54 \times 54 \times 54$ was required. In addition, the average electron-phonon coupling constant $\lambda$, which quantifies the coupling strength as well as the Allen-Dynes characteristic phonon frequency $\omega_{l n}$ [44], are related to the Eliashberg function as

$$
\begin{gathered}
\lambda=2 \int_{0}^{\infty} d \omega \frac{\alpha^{2} F(\omega)}{\omega}=\frac{1}{2 \pi \hbar N(0)} \sum_{\vec{q} \nu} \frac{\gamma_{\vec{q} \nu}}{\omega_{\vec{q} \nu}^{2}} \\
\omega_{l n}=\exp \left\{\frac{2}{\lambda} \int_{0}^{\infty} d \omega \frac{\alpha^{2} F(\omega)}{\omega} \ln \omega\right\} .
\end{gathered}
$$

Regarding $T_{c}$, it was estimated for each case by solving numerically the isotropic Migdal-Eliashberg gap equations [45-47], using the respective $\alpha^{2} F(\omega)$ for each content $x$ at its specific applied pressure value, and treating the Coulomb pseudopotential as an adjusted parameter.

Furthermore, we used the formalism of Rainer and Culleto for the calculation of the differential isotope effect coefficient $[48,49], \beta(\omega)$, to gain more insight into the coupling and how a specific phonon-frequency interval contributes to $T_{c} . \beta(\omega)$ is defined as

$$
\beta(\omega) \equiv R(\omega) \alpha^{2} F(\omega),
$$

where $R(\omega)$ is given by

$$
R(\omega)=\frac{d}{d \omega}\left[\frac{\omega}{2 T_{c}} \frac{\delta T_{c}}{\delta \alpha^{2} F(\omega)}\right]
$$




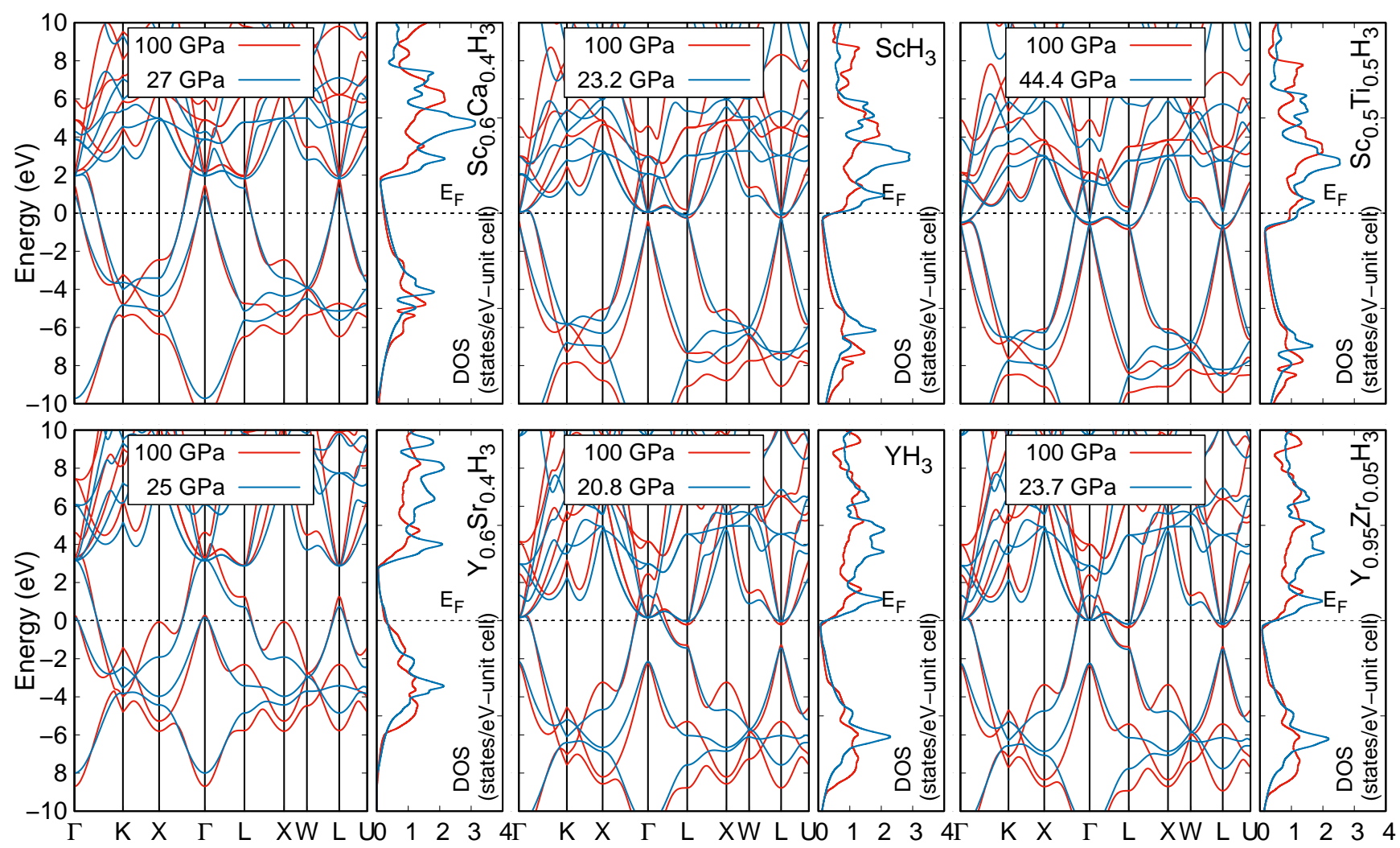

FIG. 4. Electronic band structure and density of states (DOS), for $\mathrm{Sc}_{1-x} \mathrm{M}_{x} \mathrm{H}_{3}$ and $\mathrm{Y}_{1-x} \mathrm{M}_{x} \mathrm{H}_{3}$ for the pristine $(x=0)$, the threshold electron- $(\mathrm{M}=\mathrm{Ti}, \mathrm{Zr})$ and hole- $(\mathrm{M}=\mathrm{Ca}, \mathrm{Sr})$ content, each of them at the minimum pressure where the system is dynamically stable and the maximum pressure considered.

and the $T_{c}$ functional derivative respect to the Eliashberg function is expressed as $[46,47,49,50]$

$$
\frac{\delta T_{c}}{\delta \alpha^{2} F(\omega)}=-\left.\left(\frac{d \rho}{d T}\right)\right|_{T_{c}} \frac{\delta \rho}{\delta \alpha^{2} F(\omega)}
$$

with $\rho$ corresponding to the breaking parameter that becomes zero at $T_{c}$. Finally, the isotope effect coefficient, $\alpha$, in a specific frequency interval is given by

$$
\alpha=\int_{\omega_{1}}^{\omega_{2}} d \omega \beta(\omega)
$$

\section{RESULTS AND DISCUSSION}

\section{A. Structural properties}

We performed structural optimizations of the fcc (cubic $\mathrm{NaCl}(\mathrm{B} 1))$ structure $(F m \overline{3} m$ space group) with a primitive cell of four atoms (one metal and three hydrogen) for the two $\mathrm{Sc}_{1-x} \mathrm{M}_{x} \mathrm{H}_{3}(\mathrm{M}=\mathrm{Ca}, \mathrm{Ti})$ and $\mathrm{Y}_{1-x} \mathrm{M}_{x} \mathrm{H}_{3}$ $(\mathrm{M}=\mathrm{Sr}, \mathrm{Zr})$ solid solutions at different values of metal $\mathrm{M}$ content $x$. For both systems, the equations of state were constructed from the minimum pressure, at which each system becomes stable at the cubic structure, to $100 \mathrm{GPa}$ as maximum pressure value.

For $\mathrm{Sc}_{1-x} \mathrm{M}_{x} \mathrm{H}_{3}$, the equation of state was determined for concentrations up to $x=0.5(0.4)$ of electron(hole) doping, while for $\mathrm{Y}_{1-x} \mathrm{M}_{x} \mathrm{H}_{3}$, the range was for concentrations up to $x=0.05(0.4)$ of electron(hole) doping. Electron- and hole-doping thresholds and the minimum pressures where the systems are stable were determined through dynamical instabilities, observed as imaginary frequencies in the phonon dispersion for larger $x$ contents and smaller pressure values. Phonon instabilities in metal hydrides induced by alloying have been reported previously in literature [30, 51, 52], where such dynamical behavior have been related to an increase of the heat of formation, meaning that the solid solutions become less stable.

In Fig. 2, we show the evolution of the equation of state of each metal content $x$. For both systems, $\mathrm{Sc}_{1-x} \mathrm{M}_{x} \mathrm{H}_{3}$ and $\mathrm{Y}_{1-x} \mathrm{M}_{x} \mathrm{H}_{3}$, increasing the electron content leads to a monotonous reduction of the volume, at a given pressure, as well as an increment in the minimum stable pressure value. For hole doping, the volume tendency is opposite, while the minimum stable pressure values are not following any specific trend. This behavior indicates a strengthening of the chemical bonding as the electroncontent is increased, given by the increment of $\mathrm{Zr}-$ and 
Ti-content, suggesting a hardening of phonon frequencies. Similarly, as the hole-content grows, by the increase of Sr- and Ca-content, the chemical bonding gets weaker, implying a softening of the phonon frequencies. A complete set of structural parameters, that is, the equilibrium volume $\left(V_{0}\right)$, bulk modulus $\left(B_{0}\right)$, and its pressure derivative $\left(B_{0}^{\prime}\right)$ are given in Tab. I (Appendix A), for both systems, of all $\mathrm{M}$ content $(x)$ values studied. It is worth noting that our $\mathrm{YH}_{3}$ results are in excellent agreement with the experimental data reported by Machida et al.[53].

In Fig. 3 we show the calculated cohesive energy $\left(E_{c o h}\right)$ of the two systems, within their respective electron and hole stability-range at their corresponding minimum pressure threshold. This quantity is used to characterize the stability of alloys and solid solutions, and is given by the following:

$$
E_{c o h}=E_{s y s}^{t o t}-(1-x) E_{N}^{a}-x E_{M}^{a}-3 E_{H}^{a},
$$

where $E_{\text {sys }}^{\text {tot }}$ is the total energy of the $\mathrm{N}_{1-x} \mathrm{M}_{x} \mathrm{H}_{3}$ solid solution at content $x$, while $E_{N}^{a}, E_{M}^{a}$, and $E_{H}^{a}$ are the calculated total energies of the isolated atoms $\mathrm{N}=\mathrm{Y}, \mathrm{Sc}$; $\mathrm{M}=\mathrm{Sr}, \mathrm{Zr}, \mathrm{Ca}, \mathrm{Ti}$; and hydrogen, respectively. In general, for the two solid solutions, the hole-doped systems are less stable than their corresponding pristine systems $(x=0)$ (the larger the $E_{c o h}$ absolute value, the more stable the system is), nevertheless, they are still in the stability range (negative $E_{c o h}$ ). For the case of electrondoped regime, while $\mathrm{Y}_{1-x} \mathrm{Zr}_{x} \mathrm{H}_{3}$ follows the same observed tendency than the hole-doped systems, we found that $\mathrm{Sc}_{1-x} \mathrm{Ti}_{x} \mathrm{H}_{3}$ is more stable than the pristine one, indicating the possibility to synthesize experimentally such solid solutions.

With the optimized lattice parameters and the corresponding equation of state for each system, at different content for their electron- and hole-doping regions, we proceeded to calculate their electronic and lattice dynamical properties for different applied pressure values at each $x$. Furthermore, we are presenting results obtained by the ZPE scheme. While the ZPE effects on the electronic properties are hardly visible, comparing with the static scheme, on the lattice dynamical ones, there is a noticeable softening as a general effect. This tendency comes mainly from the unit cell expansion as the ZPE contribution to the energy is taken into account.

\section{B. Electronic properties}

The evolution of the electronic band structure and the density of states at the Fermi level, $N(0)$, is analyzed in order to evaluate the effects of electron- and hole-content and pressure on the electronic properties of the solidsolutions.

In Fig. 4 we show the band structure for $\mathrm{Sc}_{1-x} \mathrm{M}_{x} \mathrm{H}_{3}$ and $\mathrm{Y}_{1-x} \mathrm{M}_{x} \mathrm{H}_{3}$ of the pristine system and of their corresponding threshold electron- $(\mathrm{Ti}, \mathrm{Zr})$ and hole- $(\mathrm{Ca}, \mathrm{Sr})$

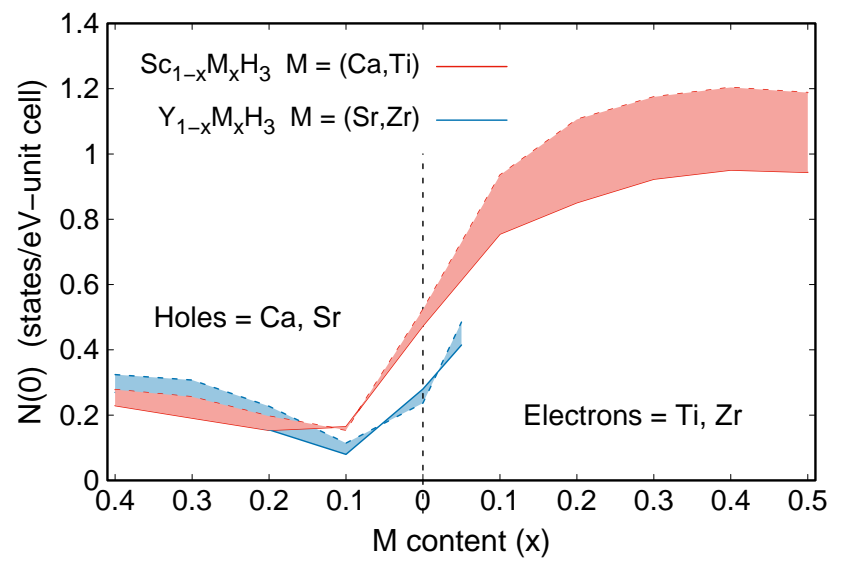

FIG. 5. Evolution of the total density of states at the Fermi level, $N(0)$, for $\mathrm{Sc}_{1-x} \mathrm{M}_{x} \mathrm{H}_{3}$ and $\mathrm{Y}_{1-x} \mathrm{M}_{x} \mathrm{H}_{3}$ as a function of the $\mathrm{M}$ content $x$ spanning the range of studied applied pressure.

doping content, each of them at the minimum pressure (where the system is dynamically stable) and the maximum pressure considered (100 GPa).

It can be seen that the main pressure effect on the band structure, independent of the electron- or holecontent, is a slight increase of the bandwidth. That is, the low-energy bands are push to even lower energy, and the high-energy ones to higher values, while keeping almost unaffected the bands around the Fermi level $\left(E_{F}\right)$. The band structure of the pristine $\mathrm{ScH}_{3}$ shows a threefold degenerate state on the L-point (giving place to one hole-like and two electron-like bands) and a twofold degenerate state on the $\Gamma$-point (electron-like bands), both located at $E_{F}$. In comparison, the pristine $\mathrm{YH}_{3}$ compound shows a twofold state on the L-point, also at the Fermi level, while the twofold state on the $\Gamma$-point is located just above $E_{F}$. As the electron content is increased for $\mathrm{ScH}_{3}$ (by Ti), the degeneracy at the L-point breaks, giving place to a new twofold state that continue to move far away from $E_{F}$, rising as an electron-like band and a hole-like band. Instead, at $\Gamma$-point the twofold state is intact, moving as a whole to lower energy values, indicating that electronic topological transitions (ETT) take place. In the case of electron-doping for $\mathrm{YH}_{3}$ (by $\mathrm{Zr}$ ), the degenerate states at $\mathrm{L}$ - and $\Gamma$-points are maintained, while they undergo a minor shift to lower energies. Regarding the hole-content increase on both systems, $\mathrm{SH}_{3}$ by $\mathrm{Ca}$ and $\mathrm{YH}_{3}$ by $\mathrm{Sr}$, its essential effect is the shift of the band structure to higher energies, without noticeable changes on the degenerate states discussed before.

Analyzing the evolution of $N(0)$, as a function of $\mathrm{M}$ content $(x)$ for the minimum and maximum pressure values of both systems, $\mathrm{Sc}_{1-x} \mathrm{M}_{x} \mathrm{H}_{3}$ and $\mathrm{Y}_{1-x} \mathrm{M}_{x} \mathrm{H}_{3}$ (see Fig. 5), it can be observed that, in general, $N(0)$ reduces at $x=0.1$ on the hole-doping regime, and in a more drastic way for the $\mathrm{ScH}_{3}$ case. As the hole-content increases, $N(0)$ starts to rise slowly, getting close to its value at $x=0$ for the $\mathrm{YH}_{3}$ system. For the electron doping 


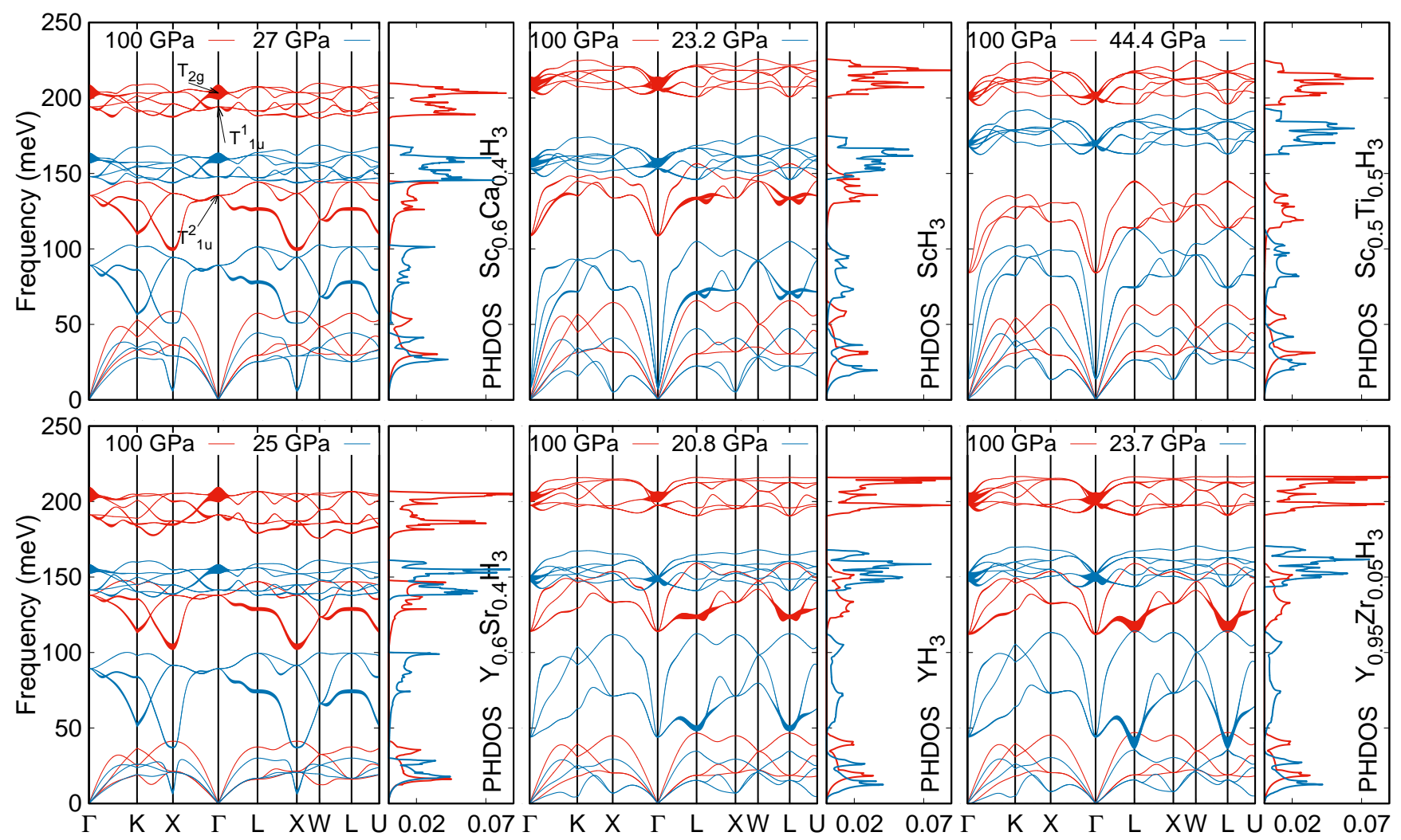

FIG. 6. Phonon dispersion, linewidths (as vertical lines along the phonon branches) and PHDOS for $\mathrm{Sc}_{1-x} \mathrm{M}_{x} \mathrm{H}_{3}$ and $\mathrm{Y}_{1-x} \mathrm{M}_{x} \mathrm{H}_{3}$ at the pristine and threshold electron- $(\mathrm{Ti}, \mathrm{Zr})$ and hole- $(\mathrm{Ca}, \mathrm{Sr})$ content, each of them at their corresponding limit pressure values.

regime, while $\mathrm{YH}_{3}$ undergoes a minor increase, mainly by the threshold limit on the $\mathrm{Zr}$-content, $\mathrm{N}(0)$ of $\mathrm{ScH}_{3}$ grows rapidly, doubling its pristine value at $x(\mathrm{Ti})=0.3$. As $x$ increases even more, the $N(0)$ grow ratio slows considerably, tending to saturate. As the applied pressure raises, $N(0)$ shows an small reduction, which is due to the expand of the bandwidth discussed previously.

\section{Lattice dynamics}

The phonon dispersion is presented on Fig. 6, including their respective phonon linewidth $\gamma_{\vec{q} \nu}$ and the phonon density of states (PHDOS), for $\mathrm{Sc}_{1-x} \mathrm{M}_{x} \mathrm{H}_{3}$ and $\mathrm{Y}_{1-x} \mathrm{M}_{x} \mathrm{H}_{3}$ at the pristine $(x=0)$ and the threshold electron- (Ti,Zr) and hole- $(\mathrm{Ca}, \mathrm{Sr})$ contents. In general, for both systems, the optical branches soften slightly as the hole-content increases, while they are shifted to higher frequencies as the electron-content rises. In particular for the high-frequency optical branches, while they shift almost on a rigid way above the frequencies of the pristine systems for the electron-doping case, on the holedoping solid-solutions they show, in addition to the softening, a small renormalization in the $T_{2 g}$ and $T_{1 u}^{1}$ optical branches at $\Gamma$. While the mid-frequency region shows subtle renormalizations at $\Gamma$ - and L-point for electroncontent regime, they are stronger for the same high- symmetry points, in addition to the X-point, for holecontent systems. Regarding the acoustic branches, they remain almost unchanged for electron-doping, whereas for hole-doping they lightly harden. Interestingly, the phonon linewidths $\gamma_{\vec{q} \nu}$ (vertical lines along the phonon branches), mainly localized around $\Gamma$ at the $T_{2 g}$ and $T_{1 u}^{1}$ optical phonon branches for $x=0$, remain almost unchanged for the hole-doping regime in both alloys, while for the electron-doping regime it slightly reduces in $\mathrm{Sc}_{1-x} \mathrm{M}_{x} \mathrm{H}_{3}$, and increases in $\mathrm{Y}_{1-x} \mathrm{M}_{x} \mathrm{H}_{3}$. In general, for both solid solid-solutions, independent of doping-scheme, the observed effect of applied pressure is a rigid hardening of the phonon frequencies and a lifting of phonon anomalies, that leans to instabilities at $\mathrm{K}$ and $\mathrm{X}$-point, for the acoustic branches.

\section{Electron-phonon and superconducting properties}

With the electronic and the lattice dynamics information, the electron-phonon spectral functions $\alpha^{2} F(\omega)$ were calculated for the entire range of hole- and electroncontent stable regimes in a broad pressure range. The $\alpha^{2} F(\omega)$ for the threshold electron- and hole-content, as well as the pristine cases, at the minimum pressure where the systems are dynamically stable, can be seen in Fig. 


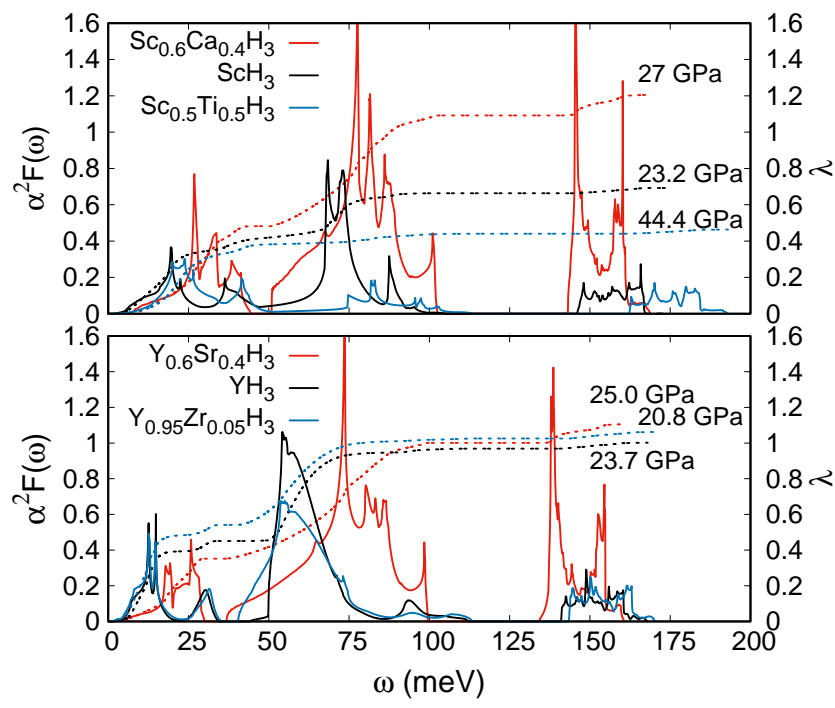

FIG. 7. Eliashberg function and partial integrated electronphonon coupling parameter $\lambda(\omega)$ for $\mathrm{Sc}_{1-x} \mathrm{M}_{x} \mathrm{H}_{3}$ and $\mathrm{Y}_{1-x} \mathrm{M}_{x} \mathrm{H}_{3}$ at $x=0$ and at the threshold electron- and holecontent for each solid-solution at the minimum pressure where the systems are dynamically stable.

7. As the electron(hole)-content increases on both systems, the high-frequency optical region of the Eliashberg function shifts to higher(lower) frequencies, while the mid-range frequency region shows an opposite behavior, and the acoustical one practically does not shift. For both systems, the weight of the spectral function is incremented as the doping goes from the electron- to the hole-content thresholds, passing through the pristine one $(x=0)$. Regarding the pressure effects, in general, for all different $x$ content cases (electron- and hole-doping), at both solid-solutions, the spectra shift to a higher frequency region as the pressure arises, going from the minimum dynamically stable value up to $100 \mathrm{GPa}$.

As the Eliashberg spectral function determines the electron-phonon coupling parameter $\lambda$ (see Eq. 3), the $\alpha^{2} F(\omega)$ observed shift due to both, doping and applied pressure, has an impact on $\lambda$ as well. The evolution of the electron-phonon coupling constant as a function of frequency, $\lambda(\omega)$, is shown in Fig.7. For the pristine $(x=0)$ and the electron-doped $\mathrm{Sc}_{1-x} \mathrm{Ti}_{x} \mathrm{H}_{3}$ solid solution, it can be observed that the main contribution to $\lambda$ comes from the acoustic region. However, for the holedoped $\mathrm{Sc}_{1-x} \mathrm{Ca}_{x} \mathrm{H}_{3}$, the main contribution comes from the mid-range frequency optical phonons. The behavior of $\lambda(\omega)$ for the $\mathrm{Y}_{1-x} \mathrm{M}_{x} \mathrm{H}_{3}$ solid solution is slightly different. In this case, both regions, the acoustic and the mid-range frequency optical ones, contribute almost at the same rate to $\lambda(\omega)$. It is worth noting that, for both solid-solutions, the high-frequency optical phonons have marginal contribution to $\lambda$.

In order to analyze the behavior of $\lambda$ as a function of pressure and electron- or hole-content for the solidsolutions, we present it as a color-map plot in Fig. 8. In

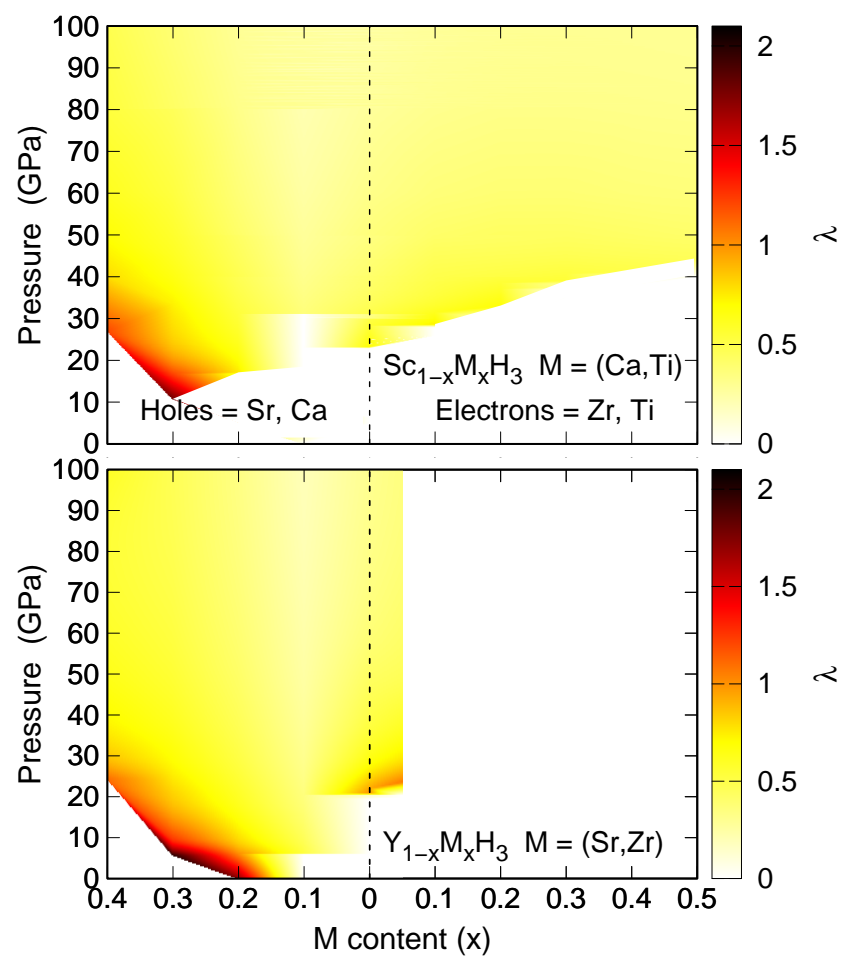

FIG. 8. Electron-phonon coupling constant $(\lambda)$ of $\mathrm{Sc}_{1-x} \mathrm{M}_{x} \mathrm{H}_{3}$ and $\mathrm{Y}_{1-x} \mathrm{M}_{x} \mathrm{H}_{3}$ for the entire studied range of electron- and hole-content and applied pressure for each solid-solution.

general, it can be observed that for most of the scanned pressure values, on both doping schemes (electron and hole) $\lambda$ hardly goes beyond 1, regardless of the solidsolution. It is worth to mention that higher $\lambda$ values are obtained for pressure close to dynamical instabilities at each electron- or hole-threshold content. Interestingly, only for very specific combination of pressure and holecontent it is possible to reach higher $\lambda$ values, like 1.8 for $\mathrm{Sc}_{0.7} \mathrm{Ca}_{0.3} \mathrm{H}_{3}$ at $10.8 \mathrm{GPa}$, and 2.0 for $\mathrm{Y}_{0.7} \mathrm{Sr}_{0.3} \mathrm{H}_{3}$ at $5.8 \mathrm{GPa}$. In particular for the latter solid-solution, the region of pressure and hole-content that could provide $\lambda$ values close to 2 is spread between $x=0.2$ and 0.3 , and around 1 to $6 \mathrm{GPa}$ on applied pressure.

In a similar fashion as $\lambda$, the evolution of the AllenDynes characteristic phonon frequency, $\omega_{l n}$, as a function of pressure and electron- and hole-content is shown in Fig. 9. It can be observed a hardening as the pressure increases, specially noticeable for the hole-doping regime and more subtle for the electron-doping one, while the lower $\omega_{l n}$ values are located at the pressure and holecontent regions where both solid-solutions present their maximum $\lambda$ values.

The calculated electron-phonon coupling properties were used to obtain estimates for the superconducting critical temperature, $T_{c}$, as a function of applied pressure and content $x$ for both solid solutions. The isotropic Migdal-Eliashberg gap equations where solved numerically with two different values of the Coulomb pseudopo- 


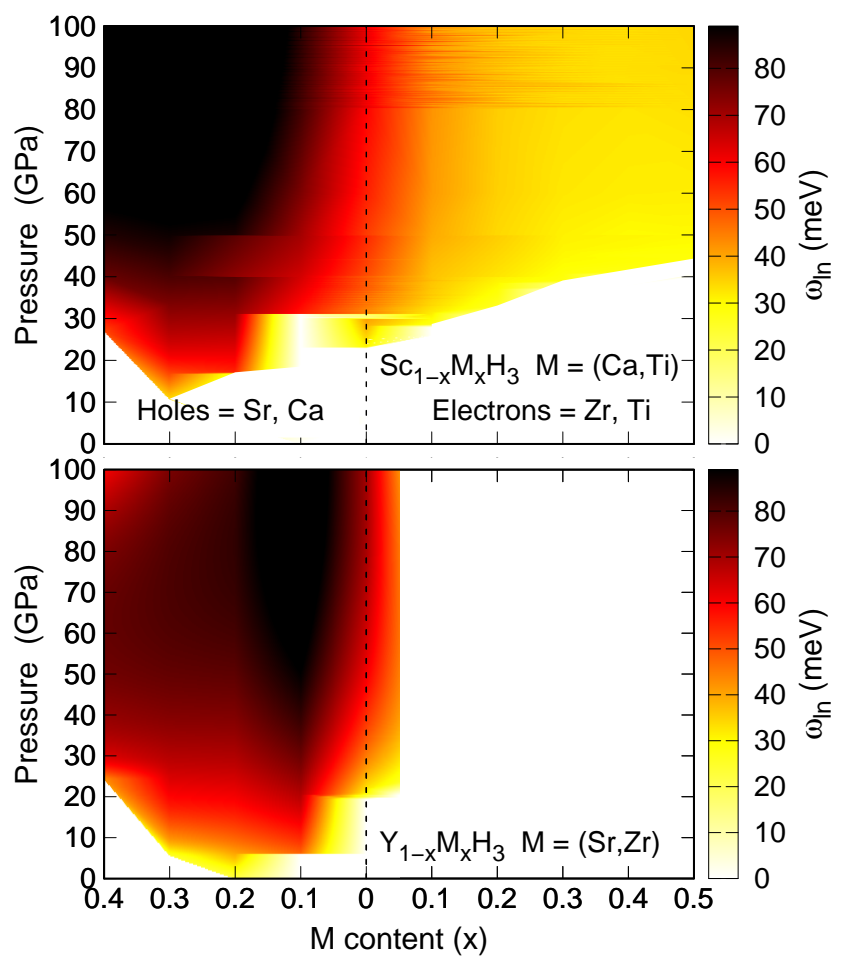

FIG. 9. Allen-Dynes characteristic phonon frequency $\left(\omega_{l n}\right)$ for $\mathrm{Sc}_{1-x} \mathrm{M}_{x} \mathrm{H}_{3}$ and $\mathrm{Y}_{1-x} \mathrm{M}_{x} \mathrm{H}_{3}$ of the complete range of electronand hole-content and applied pressure for each solid solution.

tential $\left(\mu^{*}\right): \mu^{*}=0$ (which provides an upper limit for $T_{c}$ ) and 0.15 , in order to get an idea of how strong $T_{c}$ could be affected by the $\mu^{*}$ variation. In general, we get the maximum $T_{c}$ at the minimum pressure values were we found dynamically stable solid solutions for each content $(x)$ of both regimes, electron- and hole-doping, as can be observed in Fig. 10. Comparing doping regimes, the hole-doping one reports the highest critical temperature, with values of $T_{c}=92.7(67.9) \mathrm{K}$ and $T_{c}=84.5(60.2) \mathrm{K}$ at $x=0.3$ for $\mathrm{Sc}_{1-x} \mathrm{Ca}_{x} \mathrm{H}_{3}$ and $\mathrm{Y}_{1-x} \mathrm{Sr}_{x} \mathrm{H}_{3}$ respectively, with $\mu^{*}=0(0.15)$. These maximum $T_{c}$ values corresponds to the highest $\lambda$, the lowest $\omega_{l n}$, and a comparatively low $N(0)$ (related to its corresponding electrondoping values). Such behavior shows that the tuning of the lattice dynamics is the path to enhance superconductivity in both systems. It is worth to mention that $\mathrm{Y}_{0.8} \mathrm{Sr}_{0.2} \mathrm{H}_{3}$, the only system that is dynamically stable at ambient pressure $(0 \mathrm{GPa})$, presents $T_{c}=65.4(44.3) \mathrm{K}$ for $\mu^{*}=0(0.15)$.

Regarding the pristine systems, we are fully aware that have not been experimental observation of superconductivity for fcc-phase $\mathrm{YH}_{3}$ and $\mathrm{SH}_{3}$ compounds. In particular, Kong et al.[14] reported no superconductivity for temperatures above $5 \mathrm{~K}$ for pure-fcc metallic $\mathrm{YH}_{3}$, at pressures values going from $40 \mathrm{GPa}$ up to $180 \mathrm{GPa}$. Then, in Fig. 11 we show our $T_{c}$ results for $\mathrm{ScH}_{3}$ and $\mathrm{YH}_{3}$, as a function of applied pressure, and from there it can be seen that for pressure values above $40 \mathrm{GPa}$,

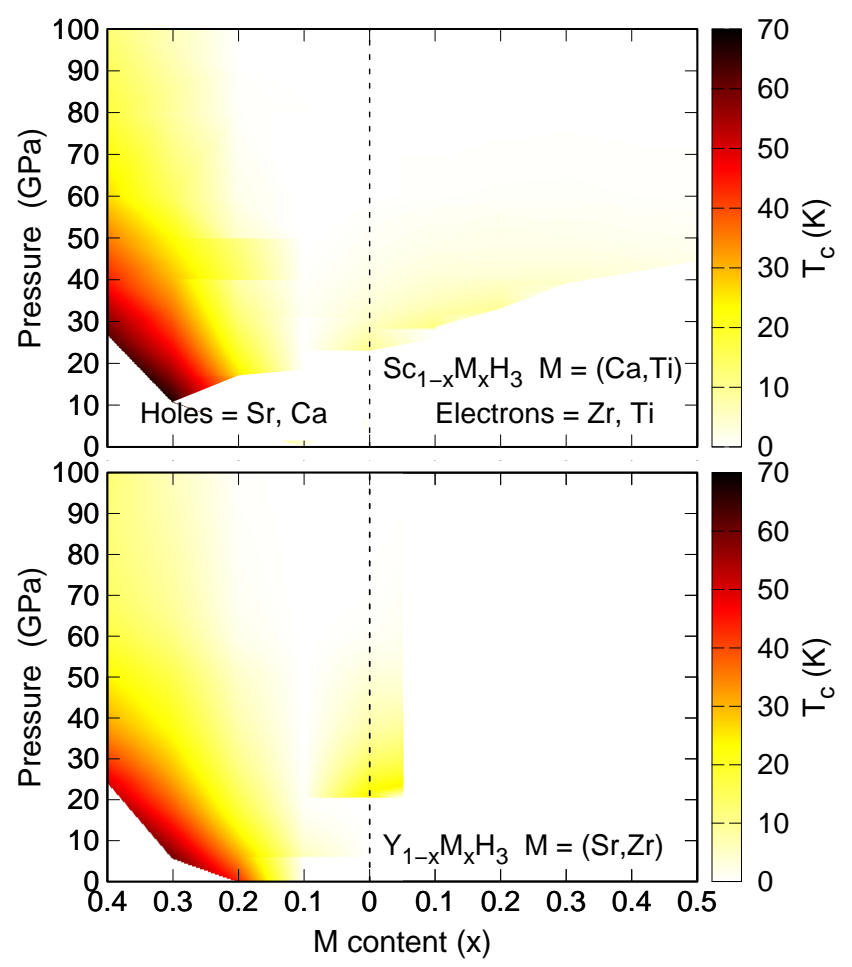

FIG. 10. Superconducting critical temperature, $T_{c}$, calculated with $\mu^{*}=0.15$, of $\mathrm{Sc}_{1-x} \mathrm{M}_{x} \mathrm{H}_{3}$ and $\mathrm{Y}_{1-x} \mathrm{M}_{x} \mathrm{H}_{3}$ at the entire range of electron- and hole-content and applied pressure for each solid-solution.

$T_{c}$ goes below $5 \mathrm{~K}$, and gets even smaller as pressure arises, in good agreement with the experimental reports. Additionally, comparing with previous results calculated by Kim et al.[28], it can be observed that the downward tendency is reproduced on both systems.

Finally, to get more insight about the contribution of the different phonon-frequency intervals to the superconductivity state, we have calculated the Rainer and Culleto differential isotope effect coefficient, $\beta(\omega)$ (Eq. 5), and the partial isotope effect $\alpha(\omega)$ (Eq. 8), which give information on how strong can $T_{c}$ be modified due to an infinitesimal ion-mass change in a specific phonon interval.

In Fig. 12 we present $\beta(\omega)$ and $\alpha(\omega)$ for both solid-solutions at the pressure and hole-content that presents the highest $T_{c}\left(\mu^{*}=0.15\right): \quad \mathrm{Sc}_{0.7} \mathrm{Ca}_{0.3} \mathrm{H}_{3}$ at $10.8 \mathrm{GPa}$ and $\mathrm{Y}_{0.7} \mathrm{Sr}_{0.3} \mathrm{H}_{3}$ at $5.8 \mathrm{GPa}$. We found $\alpha(\omega)$ to be $0.21(0.15)$ at the acoustic interval, and 0.27 (0.33) for the mid-frequency region (shadow interval) for $\mathrm{Sc}_{0.7} \mathrm{Ca}_{0.3} \mathrm{H}_{3}\left(\mathrm{Y}_{0.7} \mathrm{Sr}_{0.3} \mathrm{H}_{3}\right)$, values that contribute to the $41(30) \%$ and $53(65) \%$, respectively, of the total isotope coefficient $\alpha=0.49$, showing the importance of the mid-frequency region to the superconducting state.

In order to get an idea of how these different phonon regions contribute to $T_{c}$, we calculated the criticaltemperature change, $\Delta T_{c}$, when a phonon region is suppressed by means of the Bergmann and Rainer 


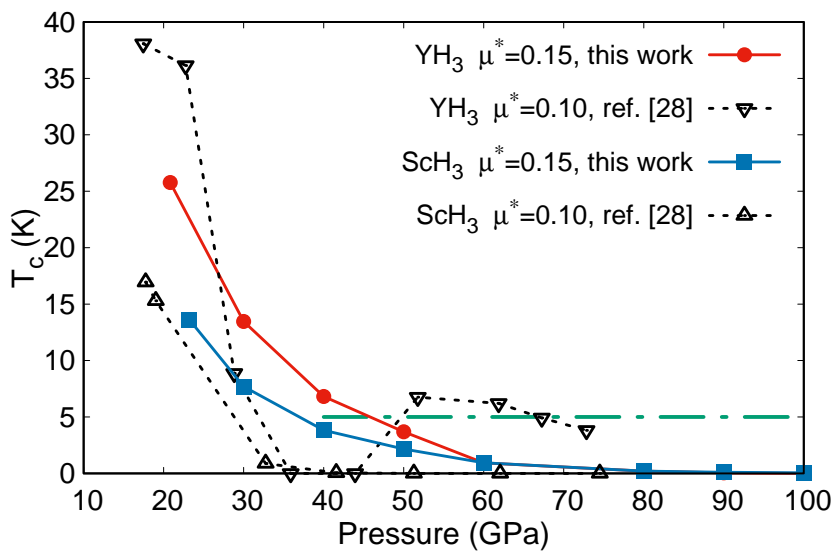

FIG. 11. Superconducting critical temperature $\left(T_{c}\right)$ calculated in this work at the entire range of applied pressure for $\mathrm{YH}_{3}$ and $\mathrm{ScH}_{3}$, compared with the calculated ones by Kim et al.[28]. The horizontal green-line represents the experimental minimal threshold temperature $(5 \mathrm{~K})$ at which Kong et al.[14] searched for superconductivity for pure-fcc metallic $\mathrm{YH}_{3}$, starting at $40 \mathrm{GPa}$.

formalism[46, 47]:

$$
\Delta T_{c}=\int_{0}^{\infty} d \omega \frac{\delta T_{c}}{\delta \alpha^{2} F(\omega)} \Delta \alpha^{2} F(\omega)
$$

where $\Delta \alpha^{2} F(\omega)=\alpha^{2} F^{\prime}(\omega)-\alpha^{2} F(\omega)$. Here, $\alpha^{2} F(\omega)$ is the total Eliashberg function and $\alpha^{2} F^{\prime}(\omega)$ is the one with a specific phonon region suppressed. Then, applying it to the same cases as before (the ones with the highest obtained $T_{c}$ for $\left.\mu^{*}=0.15\right)$ we found $\Delta T_{c}$ to be $-18.7(-11.1) \mathrm{K}$ for the acoustic region and $-52.3(-56.6) \mathrm{K}$ for the mid-frequency region for $\mathrm{Sc}_{0.7} \mathrm{Ca}_{0.3} \mathrm{H}_{3}\left(\mathrm{Y}_{0.7} \mathrm{Sr}_{0.3} \mathrm{H}_{3}\right)$, representing the later a reduction in $T_{c}$ of $77(94) \%$ when the mid-frequency region is suppressed in $\alpha^{2} F(\omega)$.

\section{CONCLUSIONS}

We have performed a thorough analysis of the structural, electronic, lattice dynamics, electron-phonon coupling, and superconducting properties of the metalhydride fcc solid-solutions $\mathrm{Sc}_{1-x} \mathrm{M}_{x} \mathrm{H}_{3}(\mathrm{M}=\mathrm{Ca}, \mathrm{Ti})$ and $\mathrm{Y}_{1-x} \mathrm{M}_{x} \mathrm{H}_{3} \quad(\mathrm{M}=\mathrm{Sr}, \mathrm{Zr})$, as a function of electron- and hole-doping content $x$, as well as applied pressure. For both systems, while increasing the electron content leads to an increment of the minimum stable pressure in comparison to pristine systems, for hole-doping we found lower minimum stable pressure values for most of content $x$, even finding a particular hole-doping case, $\mathrm{Y}_{0.8} \mathrm{Sr}_{0.2} \mathrm{H}_{3}$, that was dynamically stable at zero applied pressure. Although $N(0)$ is not improved in the whole hole-doping region for both systems, and even decreasing for $\mathrm{Sc}_{0.9} \mathrm{Ca}_{0.1} \mathrm{H}_{3}$ in comparison with $\mathrm{ScH}_{3}$,

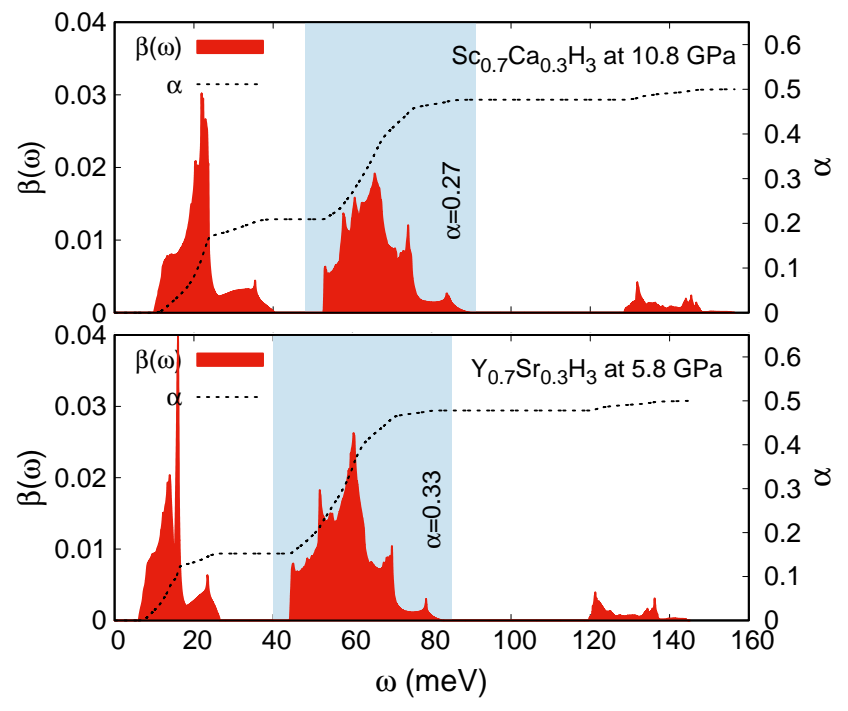

FIG. 12. Differential isotope effect coefficient $\beta(\omega)$, and partial isotope effect $\alpha(\omega)$ for both solid-solutions at the pressure and hole-content that presents the highest $T_{c}$, calculated with $\mu^{*}=0.15$.

at the electron-doping regime $N(0)$ shows an important increment at $x=0.3$ for the Sc-doped hydride, almost twice the corresponding value for the pristine system. As the pressure increases, $N(0)$ shows a reduction, which is related to the observed bandwidth expansion in both solid solutions. As for the lattice dynamics, the optical phonons soften in both systems by hole-doping, which is more pronounced for the highfrequency region than the the mid-frequency one, while the acoustic branches lightly harden. Meanwhile, for the electron-doping regime, the optical branches shift to higher values and the acoustic ones remain almost unchanged. In general, for both solid-solutions, independent of doping-scheme, the applied pressure effect is a rigid hardening of the phonon frequencies and a lifting of phonon anomalies at the acoustic branches. Regarding the electron-phonon coupling parameter, $\lambda$, the main contribution comes from the acoustic and mid-range optical phonon branches for both solid solutions, while the high-frequency optical ones have marginal participation. Interestingly, a remarkable improvement of $\lambda$ is observed in the $0.2-0.4$ hole-doping range and for low applied pressure values, close to dynamical instabilities, while by electron-doping, the systems do not improve such property, whatever value of applied pressure is considered. In particular, the systems can reach $\lambda$ values as high as 1.8 for $\mathrm{Sc}_{0.7} \mathrm{Ca}_{0.3} \mathrm{H}_{3}$ at $10.8 \mathrm{GPa}$, and 2.0 for $\mathrm{Y}_{0.7} \mathrm{Sr}_{0.3} \mathrm{H}_{3}$ at $5.8 \mathrm{GPa}$, which represent an improvement of $160 \%$ and $100 \%$, respectively, in comparison with the highest $\lambda$ values at their corresponding pristine systems, under applied pressure. Then, as a consequence, the maximum superconducting critical temperature, at each system, was obtained for those particular conditions of holedoping and pressure, with values of $T_{c}=92.7(67.9) \mathrm{K}$ 
and $T_{c}=84.5(60.2) \mathrm{K}$ for $\mathrm{ScH}_{3}$ and $\mathrm{YH}_{3}$ doped systems, respectively, with $\mu^{*}=0(0.15)$, while a general reduction of $\lambda$ and $T_{c}$ as the applied pressure rises, independent of electron- or hole-doping content, is mainly coming from the phonon hardening. Finally, by calculating the Rainer and Culleto differential isotope effect coefficient, we found the mid-frequency region as the most crucial phonon zone to the superconducting state. Then, due to all the above, we can conclude that the tuning of the lattice dynamics is a promising path for improving the superconductivity on both systems.

\section{ACKNOWLEDGMENTS}

The authors thankfully acknowledge computer resources, technical advise, and support provided by Labo- ratorio Nacional de Supercómputo del Sureste de México (LNS), a member of the CONACYT national laboratories. One of the authors (S. Villa-Cortés.) also acknowledges the Consejo Nacional de Ciencia y Tecnología (CONACyT, México) by the support under grant 769301.

\section{Appendix A: Equation of state parameters}

In Table I we show the equation of state parameters of the third-order Birch-Murnaghan equation[54] for both solid solutions, $\mathrm{Sc}_{1-x} \mathrm{M}_{x} \mathrm{H}_{3}$ and $\mathrm{Y}_{1-x} \mathrm{M}_{x} \mathrm{H}_{3}$, in the fcc $(\mathrm{NaCl}(\mathrm{B} 1))$ structure ( $F m \overline{3} m$ space group) within the ZPE scheme.
[1] N. W. Ashcroft. Hydrogen dominant metallic alloys: High temperature superconductors? Phys. Rev. Lett., 92:187002, May 2004.

[2] Lijun Zhang, Yanchao Wang, Jian Lv, and Yanming Ma. Materials discovery at high pressures. Nature Reviews Materials, 2, Feb 2017.

[3] Defang Duan, Hongyu Yu, Hui Xie, and Tian Cui. Ab initio approach and its impact on superconductivity. Journal of Superconductivity and Novel Magnetism, 32(1):5360, Jan 2019.

[4] K. Tanaka, J. S. Tse, and H. Liu. Electron-phonon coupling mechanisms for hydrogen-rich metals at high pressure. Phys. Rev. B, 96:100502, Sep 2017.

[5] Tiange Bi, Niloofar Zarifi, Tyson Terpstra, and Eva Zurek. The search for superconductivity in high pressure hydrides. In Reference Module in Chemistry, Molecular Sciences and Chemical Engineering. Elsevier, 2019.

[6] Duan Defang, Liu Yunxian, Tian Fubo, Li Da, Huang Xiaoli, Zhao Zhonglong, Yu Hongyu, Liu Bingbing, Tian Wenjing, and Cui Tian. Pressure-induced metallization of dense (h2s)2h2 with high-tc superconductivity. Scientific Reports, 4, Sept 2014.

[7] Hanyu Liu, Ivan I. Naumov, Roald Hoffmann, N. W. Ashcroft, and Russell J. Hemley. Potential high-tc superconducting lanthanum and yttrium hydrides at high pressure. Proceedings of the National Academy of Sciences, 114(27):6990-6995, 2017.

[8] Hui Wang, John S. Tse, Kaori Tanaka, Toshiaki Iitaka, and Yanming Ma. Superconductive sodalite-like clathrate calcium hydride at high pressures. Proceedings of the National Academy of Sciences, 109(17):6463-6466, 2012.

[9] A. P. Drozdov, M. I. Eremets, I. A. Troyan, V. Ksenofontov, and S. I. Shylin. Conventional superconductivity at 203 kelvin at high pressures in the sulfur hydride system. Nature, Letter, 525:73-76, Sept 2015.

[10] Mari Einaga, Masafumi Sakata, Takahiro Ishikawa, Katsuya Shimizu, Mikhail I. Eremets, Alexander P. Drozdov, Ivan A. Troyan, Naohisa Hirao, and Yasuo Ohishi. Crystal structure of the superconducting phase of sulfur hydride. Nature Physics, 12, 2016.
[11] A. P. Drozdov, P. P. Kong, V. S. Minkov, S. P. Besedin, M. A. Kuzovnikov, S. Mozaffari, L. Balicas, F. Balakirev, D. Graf, V. B. Prakapenka, E. Greenberg, D. A. Knyazev, M. Tkacz, and M. I. Eremets. Superconductivity at 250 $\mathrm{k}$ in lanthanum hydride under high pressures. Nature, 569:528-531, 2019.

[12] Maddury Somayazulu, Muhtar Ahart, Ajay K. Mishra, Zachary M. Geballe, Maria Baldini, Yue Meng, Viktor V. Struzhkin, and Russell J. Hemley. Evidence for superconductivity above $260 \mathrm{k}$ in lanthanum superhydride at megabar pressures. Phys. Rev. Lett., 122:027001, Jan 2019.

[13] Elliot Snider, Nathan Dasenbrock-Gammon, Raymond McBride, Xiaoyu Wang, Noah Meyers, Keith V. Lawler, Eva Zurek, Ashkan Salamat, and Ranga P. Dias. Synthesis of yttrium superhydride superconductor with a transition temperature up to $262 \mathrm{k}$ by catalytic hydrogenation at high pressures. Phys. Rev. Lett., 126:117003, Mar 2021.

[14] Panpan Kong, Vasily S. Minkov, Mikhail A. Kuzovnikov, Alexander P. Drozdov, Stanislav P. Besedin, Shirin Mozaffari, Luis Balicas, Fedor Fedorovich Balakirev, Vitali B. Prakapenka, Stella Chariton, Dmitry A. Knyazev, Eran Greenberg, and Mikhail I. Eremets. Superconductivity up to $243 \mathrm{k}$ in the yttrium-hydrogen system under high pressure. Nature Communications, 12, Aug 2021.

[15] Elliot Snider, Nathan Dasenbrock-Gammon, Raymond McBride, Mathew Debessai, Hiranya Vindana, Kevin Vencatasamy, Keith V. Lawler, Ashkan Salamat, and Ranga P. Dias. Room-temperature superconductivity in a carbonaceous sulfur hydride. Nature, 586:373-377, Octuber 2020.

[16] Yinwei Li, Jian Hao, Hanyu Liu, John S. Tse, Yanchao Wang, and Yanming Ma. Pressure-stabilized superconductive yttrium hydrides. Scientific Reports, 5:9948, May 2015.

[17] Xiaoqiu Ye, Niloofar Zarifi, E. Zurek, R. Hoffmann, and N. Ashcroft. High hydrides of scandium under pressure: Potential superconductors. Journal of Physical Chemistry $C, 2018$. 
TABLE I. Birch-Murnaghan fit to the equation of state. $V_{0}$ is the reference volume at zero pressure (in $a_{B}^{3}$, where $a_{B}$ is the Bohr radius), $B_{0}$ is the bulk modulus in GPa at zero pressure, and $B_{0}^{\prime}$ is the pressure derivative of the bulk modulus.

\begin{tabular}{|c|c|c|c|}
\hline \multicolumn{4}{|c|}{$\mathrm{Sc}_{1-x} \mathrm{Ca}_{x} \mathrm{H}_{3}$} \\
\hline$x$ & $V_{0}\left(a_{B}^{3}\right)$ & $B_{0}(\mathrm{GPa})$ & $B_{0}^{\prime}$ \\
\hline 0.1 & 191.10 & 100.72 & 3.66 \\
\hline 0.2 & 197.03 & 92.94 & 3.70 \\
\hline 0.3 & 202.76 & 86.40 & 3.70 \\
\hline 0.4 & 207.74 & 82.47 & 3.74 \\
\hline \multicolumn{4}{|c|}{$\mathrm{ScH}_{3}$} \\
\hline & $V_{0}\left(a_{B}^{3}\right)$ & $B_{0}(\mathrm{GPa})$ & $B_{0}^{\prime}$ \\
\hline & 189.06 & 104.49 & 3.64 \\
\hline \multicolumn{4}{|c|}{$\mathrm{Sc}_{1-x} \mathrm{Ti}_{x} \mathrm{H}_{3}$} \\
\hline$x$ & $V_{0}\left(a_{B}^{3}\right)$ & $B_{0}(\mathrm{GPa})$ & $B_{0}^{\prime}$ \\
\hline 0.1 & 184.52 & 106.24 & 3.69 \\
\hline 0.2 & 180.43 & 107.47 & 3.73 \\
\hline 0.3 & 174.96 & 116.36 & 3.68 \\
\hline 0.4 & 170.91 & 121.80 & 3.66 \\
\hline 0.5 & 169.66 & 116.31 & 3.77 \\
\hline \multicolumn{4}{|c|}{$\mathrm{Y}_{1-x} \mathrm{Sr}_{x} \mathrm{H}_{3}$} \\
\hline$x$ & $V_{0}\left(a_{B}^{3}\right)$ & $B_{0}(\mathrm{GPa})$ & $B_{0}^{\prime}$ \\
\hline 0.1 & 245.38 & 89.43 & 3.77 \\
\hline 0.2 & 251.58 & 79.36 & 3.77 \\
\hline 0.3 & 258.51 & 74.04 & 3.78 \\
\hline 0.4 & 265.01 & 70.43 & 3.77 \\
\hline \multicolumn{4}{|c|}{$\mathrm{YH}_{3}$} \\
\hline & $V_{0}\left(a_{B}^{3}\right)$ & $B_{0}(\mathrm{GPa})$ & $B_{0}^{\prime}$ \\
\hline & 240.35 & 88.21 & 3.77 \\
\hline \multicolumn{4}{|c|}{$\mathrm{Y}_{1-x} \mathrm{Zr}_{x} \mathrm{H}_{3}$} \\
\hline$x$ & $V_{0}\left(a_{B}^{3}\right)$ & $B_{0}(\mathrm{GPa})$ & $B_{0}^{\prime}$ \\
\hline 0.05 & 238.20 & 89.10 & 3.79 \\
\hline
\end{tabular}

[18] Tetsuji Kume, Hiroyuki Ohura, Shigeo Sasaki, Hiroyasu Shimizu, Ayako Ohmura, Akihiko Machida, Tetsu Watanuki, Katsutoshi Aoki, and Kenichi Takemura. High-pressure study of $\mathrm{yh}_{3}$ by raman and visible absorption spectroscopy. Phys. Rev. B, 76:024107, Jul 2007.

[19] Ayako Ohmura, Akihiko Machida, Tetsu Watanuki, Katsutoshi Aoki, Satoshi Nakano, and K. Takemura. Infrared spectroscopic study of the band-gap closure in $\mathrm{yh}_{3}$ at high pressure. Phys. Rev. B, 73:104105, Mar 2006.

[20] A. Machida, A. Ohmura, T. Watanuki, K. Aoki, and K. Takemura. Long-period stacking structures in yttrium trihydride at high pressure. Phys. Rev. B, 76:052101, Aug 2007.

[21] T. Palasyuk and M. Tkacz. Hexagonal to cubic phase transition in yh3 under high pressure. Solid State Communications, 133(7):477-480, 2005.

[22] Riki Kataoka, Toru Kimura, Nobuhiko Takeichi, and Atsunori Kamegawa. Stabilization of face-centered cubic high-pressure phase of reh3 ( $\mathrm{re}=\mathrm{y}, \mathrm{gd}$, dy) at ambient pressure by alkali or alkaline-earth substitution. Inorganic Chemistry, 57(8):4686-4692, 2018. PMID:
29620366.

[23] Riki Kataoka, Toru Kimura, Kouji Sakaki, Masashi Nozaki, Toshikatsu Kojima, Kazutaka Ikeda, Toshiya Otomo, Nobuhiko Takeichi, and Atsunori Kamegawa. Facile synthesis of lih-stabilized face-centered-cubic yh3 high-pressure phase by ball milling process. Inorganic Chemistry, 58(19):13102-13107, 2019. PMID: 31502447.

[24] J. Purans, A. P. Menushenkov, S. P. Besedin, A. A. Ivanov, V. S. Minkov, I. Pudza, A. Kuzmin, K. V. Klementiev, S. Pascarelli, O. Mathon, A. D. Rosa, T. Irifune, and Mikhail I. Eremets. Local electronic structure rearrangements and strong anharmonicity in yh3 under pressures up to 180gpa. Nature Communications, 12, March 2021.

[25] Tetsuji Kume, Hiroyuki Ohura, Tomoo Takeichi, Ayako Ohmura, Akihiko Machida, Tetsu Watanuki, Katsutoshi Aoki, Shigeo Sasaki, Hiroyasu Shimizu, and Kenichi Takemura. High-pressure study of sch3: Raman, infrared, and visible absorption spectroscopy. Phys. Rev. B, 84:064132, Aug 2011.

[26] Bo Kong, Zhu-Wen Zhou, De-Liang Chen, and RongFeng Ling-hu. Structures and phase transitions of ScH3under high pressure. Chinese Physics B, 22(5):057102, may 2013.

[27] T Pakornchote, U Pinsook, and T Bovornratanaraks. The hcp to fcc transformation path of scandium trihydride under high pressure. Journal of Physics: Condensed Matter, 26(2):025405, dec 2013.

[28] Duck Young Kim, Ralph H. Scheicher, Ho-kwang Mao, Tae W. Kang, and Rajeev Ahuja. General trend for pressurized superconducting hydrogen-dense materials. Proceedings of the National Academy of Sciences, 107(7):2793-2796, 2010.

[29] J Y Zhang, L J Zhang, T Cui, Y L Niu, Y M Ma, Z He, and G T Zou. A first-principles study of electron-phonon coupling in electron-doped LiH. Journal of Physics: Condensed Matter, 19(42):425218, sep 2007.

[30] M. A. Olea-Amezcua, O. De la Peña Seaman, and R. Heid. Superconductivity by doping in alkali-metal hydrides without applied pressure: An ab initio study. Phys. Rev. B, 99:214504, Jun 2019.

[31] S Villa-Cortés and O De la Peña-Seaman. Electron- and hole-doping on ScH2 and YH2: effects on superconductivity without applied pressure. Journal of Physics: Condensed Matter, 33(42):425401, aug 2021.

[32] W. Kohn and L. J. Sham. Self-consistent equations including exchange and correlation effects. Phys. Rev., 140:A1133-A1138, Nov 1965.

[33] Lothar Nordheim. Zur elektronentheorie der metalle. i. Annalen der Physik, 401(5):607-640, 1931.

[34] H. Rosner, A. Kitaigorodsky, and W. E. Pickett. Prediction of high $t_{c}$ superconductivity in hole-doped libc. Phys. Rev. Lett., 88:127001, Mar 2002.

[35] Lilia Boeri, Jens Kortus, and O. K. Andersen. Threedimensional $\mathrm{mgb}_{2}$-type superconductivity in hole-doped diamond. Phys. Rev. Lett., 93:237002, Nov 2004.

[36] Yanfeng Ge, Fan Zhang, and Yugui Yao. First-principles demonstration of superconductivity at $280 \mathrm{k}$ in hydrogen sulfide with low phosphorus substitution. Phys. Rev. B, 93:224513, Jun 2016.

[37] O. De la Peña Seaman, R. de Coss, R. Heid, and K.P. Bohnen. Effects of al and c doping on the electronic structure and phonon renormalization in $\mathrm{mgb}_{2}$. Phys. Rev. B, 79:134523, Apr 2009. 
[38] Paolo Giannozzi et al. Quantum espresso: a modular and open-source software project for quantum simulations of materials. Journal of Physics: Condensed Matter, 21(39):395502, 2009.

[39] Stefano Baroni, Stefano de Gironcoli, Andrea Dal Corso, and Paolo Giannozzi. Phonons and related crystal properties from density-functional perturbation theory. Rev. Mod. Phys., 73:515-562, Jul 2001.

[40] John P. Perdew, Kieron Burke, and Matthias Ernzerhof. Generalized gradient approximation made simple. Phys. Rev. Lett., 77:3865-3868, Oct 1996.

[41] Stefano Baroni, Paolo Giannozzi, and Eyvaz Isaev. Density-Functional Perturbation Theory for QuasiHarmonic Calculations. Reviews in Mineralogy and Geochemistry, 71(1):39-57, Jan 2010.

[42] Philip B. Allen. Neutron spectroscopy of superconductors. Phys. Rev. B, 6:2577-2579, Oct 1972.

[43] Philip B. Allen and Richard Silberglitt. Some effects of phonon dynamics on electron lifetime, mass renormalization, and superconducting transition temperature. Phys. Rev. B, 9:4733-4741, Jun 1974.

[44] P. B. Allen and R. C. Dynes. Transition temperature of strong-coupled superconductors reanalyzed. Phys. Rev. B, 12:905-922, Aug 1975.

[45] G. M. Eliashberg. Interaction between electrons and lattice vibrations in a superconductor. J. Exptl. Theoret. Phys., 38:966-976, 1960.

[46] G. Bergmann and D. Rainer. The sensitivity of the transition temperature to changes in $\alpha 2 \mathrm{f}(\omega)$. Zeitschrift für Physik, 263(1):59-68, 1973.

[47] S. Villa-Cortés and R. Baquero. The thermodynamics and the inverse isotope effect of superconducting pal- ladium hydride compounds under pressure. Journal of Physics and Chemistry of Solids, 123:371 - 377, 2018.

[48] D. Rainer and F. J. Culetto. Theory of the isotope effect in superconducting compounds: Pdd and $\mathrm{mo}_{6} \mathrm{se}_{8}$. Phys. Rev. B, 19:2540-2547, Mar 1979.

[49] S. Villa-Cortés and O. De la Peña-Seaman. Effect of van hove singularity on the isotope effect and critical temperature of h3s hydride superconductor as a function of pressure. Journal of Physics and Chemistry of Solids, 161:110451, 2022.

[50] S. Villa-Cortés and R. Baquero. On the calculation of the inverse isotope effect in $\mathrm{pdh}(\mathrm{d})$ : A migdal-eliashberg theory approach. Journal of Physics and Chemistry of Solids, 119:80 - 84, 2018.

[51] X. Q. Zeng, L. F. Cheng, J. X. Zou, W. J. Ding, H. Y. Tian, and C. Buckley. Influence of $3 \mathrm{~d}$ transition metals on the stability and electronic structure of mgh2. Journal of Applied Physics, 111(9):093720, 2012.

[52] Y. Song, Z. X. Guo, and R. Yang. Influence of selected alloying elements on the stability of magnesium dihydride for hydrogen storage applications: A first-principles investigation. Phys. Rev. B, 69:094205, Mar 2004.

[53] A. Machida, A. Ohmura, T. Watanuki, T. Ikeda, K. Aoki, S. Nakano, and K. Takemura. X-ray diffraction investigation of the hexagonal-fcc structural transition in yttrium trihydride under hydrostatic pressure. Solid State Communications, 138(9):436-440, 2006.

[54] F. D. Murnaghan. Finite deformations of an elastic solid. American Journal of Mathematics, 59(2):235-260, 1937. 